\title{
THEORETICAL STUDY OF OPTICAL AND XANES SPECTRA FOR CdTe WITHIN THE $k$-DEPENDENT MATRIX ELEMENT APPROACH
}

\author{
R. Markowski, J. Oleszkiewicz and A. Kisiel \\ Institute of Physics, Jagiellonian University \\ Reymonta 4, 30-059 Kraków, Poland
}

The reflectivity and X-ray absorption near-edge structure spectra for CdTe were calculated with the inclusion of the transition matrix elements from band structure obtained within self-consistent, relativistic linear muffin tin orbital method. The results were compared with experimental data for the case of CdTe and with our previous calculations.

PACS numbers: 78.70.Dm, 78.20.Bh, 71.25.Tn

The LMTO method which we used in this study of the optical properties and XANES spectra of CdTe is described elsewhere [1] and the details of its application to the calculation of the electronic structure of the zinc-blende-type compounds are presented in other papers $[2,3]$. The band structure was calculated with the inclusion of the "combined correction term" in the "frozen core approximation" [4]. LSDA potential was used in the form proposed by Vosko, Wilk and Nusair [5]. The openness of the zinc-blende structure was dealt in standard way by placing additional "empty spheres" into the unit cell. Scalar relativistic corrections were consistently applied in the self-consistent procedure. In the final band-structure calculation the spin-orbit coupling was included as a formal perturbation term in the Hamiltonian [1]. The self-consistent energy eigenvalues and wave functions were used to determine the complex dielectric functions.

The optical absorption is proportional to the imaginary part of the dielectric function $\epsilon_{2}^{b}(\hbar \omega)$ given by the following expression [6]:

$$
\epsilon_{2}^{b}(\hbar \omega)=\frac{\text { const }}{\omega^{2}} \sum_{i} \sum_{f} \int_{\mathrm{BZ}} \mathrm{d}^{3} k\left|M_{f i}^{k}\right|^{2} \delta\left(E_{f}^{k}-E_{i}^{k}-\hbar \omega\right),
$$

where $M_{f i}^{k}=\langle f k|P| i k\rangle$ is the dipole matrix element of the momentum operator. The calculations were carried out in a single energy panel using $5 s, 5 p$ and $5 d$ basis functions for Te and $5 s, 5 p$ and $4 d$ basis functions for $\mathrm{Cd}$. The finite lifetime was incorporated in the results by convoluting them with a Lorentzian function of a width that depends on the final state energy. We also took into account the experimental resolution in the Gaussian form. In general, our calculations allow for a consistent assignment of structures in the experimental reflectivity spectrum and 
for understanding the physical mechanism of the transitions observed in the $R(\omega)$ spectrum. We show (Fig. 1) that interpretation of the UV part of the spectra proposed by Cardona et al. [7] and Kisiel et al. [8] is entirely correct, when all relativistic terms are included in the calculation procedure. In papers $[2,3]$ we suggested that the structure assigned to core excitons can be described within the framework of a band-to-band transition formalism. However, the structure is removed when the convolution with a Lorentzian function is included.
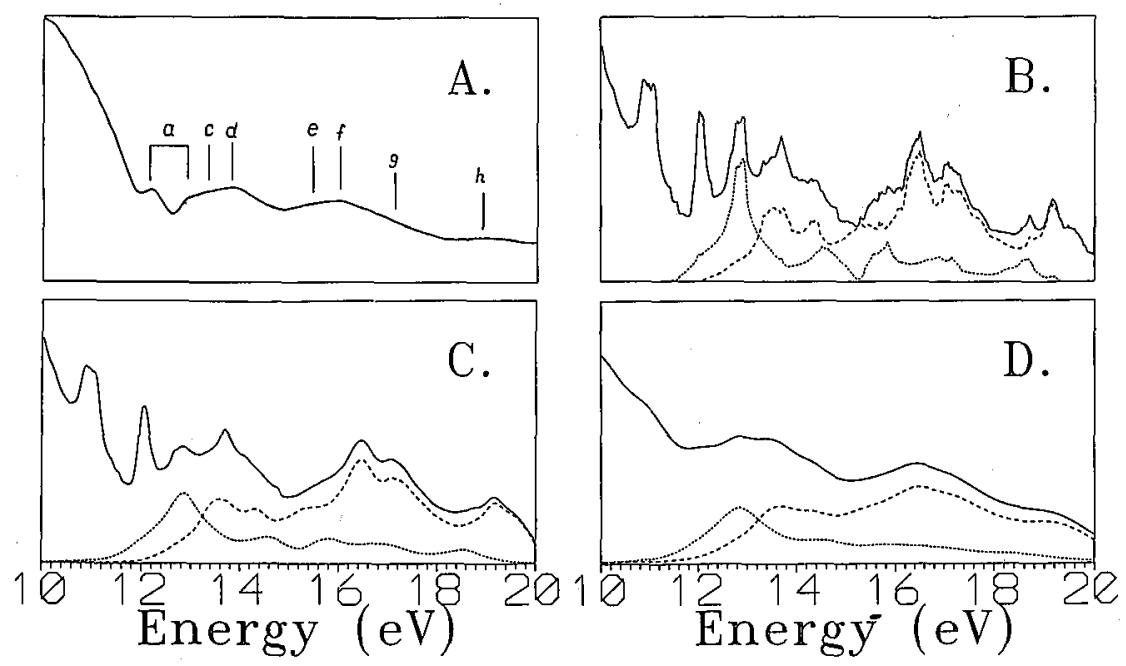

Fig. 1. Reflectivity spectrum of CdTe in the UV energy range: (A) experimental spectrum measured at room temperature in the region of the cation $d$ excitations [8]; (B) theoretical "raw" result: the broken curve (short dashes) represents the contribution of band 1, the broken curve (long length dashes) the contribution of bands 2 to 6 , the full curve represents the total absorption spectrum; (C) theoretical "convoluted" result: $\Gamma_{\mathrm{L}}$ does not depend on the final state energy; (D) theoretical "convoluted" result: $\Gamma_{L}$ depends on the final state energy [11].

In the one-electron approximation the X-ray absorption coefficient from a core state $|c\rangle$ with energy $E_{c}$ to final unoccupied conduction states $|f k\rangle$ with energy $E_{f}^{k}$ is given by the expression [6]:

$$
\mu_{0}(\hbar \omega)=\text { const } \sum_{f} \int_{\mathbf{B Z}} \mathrm{d}^{3} k\left|M_{f c}^{k}\right|^{2} \delta\left(E_{f}^{k}-E_{c}-\hbar \omega\right),
$$

where $M_{f i}^{k}=\langle f k|\boldsymbol{P}| c\rangle$ is the dipole transition matrix element. The core states were calculated by self-consistent Dirac-Slater method on the basis of modified Liberman program [9]. The final conduction states have finite lifetime, therefore the Müller correction was taken into account [10]. The absorption coefficient was finally convoluted with Gaussian and Lorentzian functions. The value of Lorentzian width $\Gamma_{\mathrm{L}}$ was optimized to obtain the best agreement of the theoretical spectrum 
with the experimental one in the surrounding of the absorption edge (about $8 \mathrm{eV}$ around the edge first inflection point). The calculation was carried out in two energy panels using $6 s, 5 p, 5 d$ basis functions for Te and $5 s, 5 p, 5 d$ basis functions for Cd.

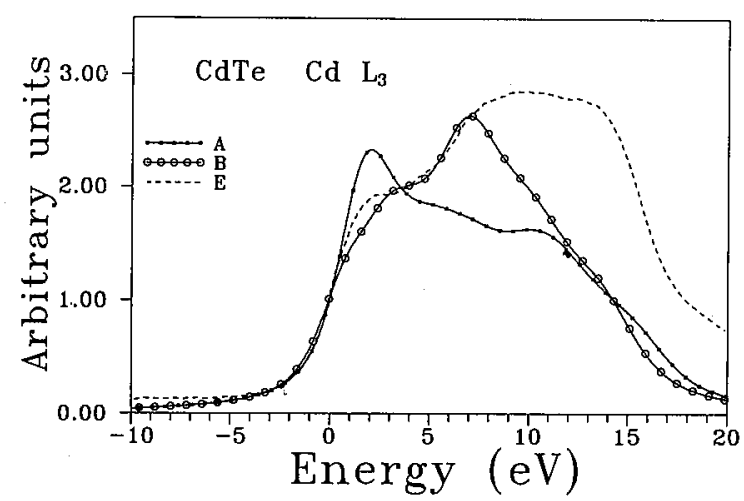

Fig. 2. The calculated Cd $L_{3} \mathrm{X}$-ray edge spectra for CdTe. The curve - the calculations with inclusion of dipol transition matrix elements and Cd $4 d$ basis functions. The curve $B$ - the calculations with inclusion of dipol transition matrix elements and Cd $5 d$ basis functions. The curve $E-$ experimental spectrum.

From comparison of the theoretical results with experimental data it follows that the $\mathrm{Cd} L_{1}$ and $\mathrm{Te} L_{1}$ theoretical X-ray absorption edges results give almost equivalent good description of the experimental spectra [11]. Figure 2 presents the results for the $\mathrm{Cd} L_{3} \mathrm{X}$-ray absorption edge which arises from superposition of the transitions to $s$-like and $d$-like conduction states. The worst agreement between theory and experiment exists for $\mathrm{Cd} L_{3}$ edge when the basis function set includes the $4 d \mathrm{Cd}$ functions but does not include the $5 d \mathrm{Cd}$ functions $[6,11]$. When the basis function set contains $5 d$ Cd functions, the agreement between theory and experiment is much better.

\section{References}

[1] O.K. Andersen, Phys. Rev. B 12, 3060 (1975).

[2] R. Markowski, M. Podgórny, J. Phys. Condens. Matter 3, 9041 (1991).

[3] R. Markowski, M. Podgórny, J. Phys. Condens. Matter 4, 2505 (1992).

[4] H.L. Skriver, The LMTO Method, Vol. 41 of Solid State Sciences, Springer, Berlin 1984.

[5] S.H. Vosko, L. Wilk, M. Nusair, Can. J. Phys. 58, 1200 (1980).

[6] R. Markowski, J. Oleszkiewicz, A. Kisiel, Acta Phys. Pol. A 80, 369 (1991).

[7] M. Cardona, D.L. Greenaway, Phys. Rev. B 4, 451 (1963).

[8] A. Kisiel, M. Zimnal-Starnawska, F. Antonangeli, M. Piacentini, N. Zema, Nuovo Cimento D 8, 436 (1986). 
[9] D. Liberman, J.T. Waber, D.T. Cromer, Phys. Rev. 137, 27 (1965).

[10] J.E. Müller, O. Jepsen, J.W. Wilkins, Solid State Commun. 42, 365 (1982).

[11] A. Kisiel, G. Dalba, P. Fornasini, M. Podgórny, J. Oleszkiewicz, F. Rocca, E. Burattini, Phys. Rev. B 39, 7895 (1989). 